\title{
Acknowledgement of Reviewers
}

We gratefully acknowledge the work of reviewers for Journal of Plant Diseases and Protection. The standards of scientific publishing are upheld by peer review and the work of our reviewers. We thank the many scientists who have given of their time and expertise to offer their judgements and to help authors in improving manuscripts during the year 2015.

Catherine Abadie, Capesterre-Belle-Eau, Guadeloupe

David Backhouse, Armidale, Australia

Yolanda Bel, Valencia, Spain

Regina Belz, Hohenheim, Germany

Anna Berlin, Uppsala, Sweden

Michael Birkett, Harpenden, UK

Charles Cernay, Thiverval-Grignon, France

Stéphane Compant, Tulln, Austria

Sylvain Cordelier, Reims, France

Abdelfattah A Dababat, Ankara, Turkey

Regine Delourme, Rennes, France

Moussa El Jarroudi, Arlon, Belgium

Hugh Evans, Aberystwyth, UK

Elisabete Figueiredo, Lisboa, Portugal

Christoph Flucke, Rostock, Germany

Sylvain Fournet, Rennes, France

M Stella Grando, San Michele all 'Adige, Italy

Laura Grenville-Briggs Didymus, Alnarp, Sweden

Matthias Hahn, Kaiserslautern, Germany

Pavel Hamouz, Prague, Czech Republic

Jianju J Hao, Orono, Maine, USA

Fredrik Heyman, Uppsala, Sweden

Matthieu Joosten, Wageningen, The Netherlands

George Karaoglanidis, Thessaloníki, Greece

Corné Kempenaar, Wageningen, The Netherlands

Nabil Killiny, Gainesville, FL, USA

Noel L Knight, Toowoomba, Australia

Harri Kokko, Kuopio, Finland

Andreas Kollar, Dossenheim, Germany

Birger Koopmann, Göttingen, Germany

Evsey Kosman, Tel Aviv, Israel

Per Kudsk, Aarhus, Denmark

Vicente Medina Piles, Lleida, Spain

Lucie Michel, Thiverval-Grignon, France
Gerald Moritz, Halle, Germany

Vicente Navarro-Llopis, València, Spain

Jan Nechwatal, Freising, Germany

Lise Nistrup Jørgensen, Aarhus, Denmark

Erich-Christian Oerke, Bonn, Germany

Feyisara Olorunleke, Ghent, Belgium

Euro Pannacci, Perugia, Italy

Dong Suk Park, Suwon, Korea

Nuria Pedrol, Vigo, Spain

Jan Petersen, Bingen, Germany

Julie A Peterson, Lincoln, USA

Hans-Michael Poehling, Hannover, Germany

Jarrad R Prasifka, Fargo, USA

Mohammad Razavi, Tehran, Iran

Annette Reineke, Geisenheim, Germany

Katja Richert-Pöggeler, Braunschweig, Germany

Nadine Riediger, Göttingen, Germany

Vittorio Rossi, Piacenza, Italy

Christoph Schmidt, Průhonice, Czech Republic

Bernd Schneider, Dossenheim, Germany

Corrie Schomaker, Wageningen, The Netherlands

Francesco Sorribas, Barcelona, Spain

Arne Stensvand, As, Norway

Anurag Sunpapao, Hat Yai, Thailand

Mark Sutherland,Toowoomba, Australia

Patricia Tamez Guerra, San Nicholás, Mexico

Krzysztof Treder, Bonin, Poland

Susanne Vogelgsang, Zürich, Switzerland

Anne-Sophie Walker, Versailles, France

Roland Weber, Jork, Germany

Andreas Westphal, Parlier, CA, USA

Mark Winter, Göttingen, Germany

Yufan Zhang, Pennsylvania State University, PA, USA

Eva Zyprian, Geilweilerhof, Germany 